\title{
New Exact Solutions for a Higher Order Wave Equation of KdV Type Using Multiple $G^{\prime} / G$-Expansion Methods
}

\author{
Yinghui He \\ Department of Mathematics, Honghe University, Mengzi, Yunnan 661100, China \\ Correspondence should be addressed to Yinghui He; heyinghui07@163.com
}

Received 16 February 2014; Accepted 9 April 2014; Published 29 April 2014

Academic Editor: Giorgio Kaniadakis

Copyright ( 2014 Yinghui He. This is an open access article distributed under the Creative Commons Attribution License, which permits unrestricted use, distribution, and reproduction in any medium, provided the original work is properly cited.

\begin{abstract}
The $G^{\prime} / G$-expansion method is a powerful mathematical tool for solving nonlinear wave equations in mathematical physics and engineering problems. In our work, exact traveling wave solutions of a generalized KdV type equation of neglecting the highest order infinitesimal term, which is an important water wave model, are discussed by the $G^{\prime} / G$-expansion method and its variants. As a result, many new exact solutions involving parameters, expressed by Jacobi elliptic functions, hyperbolic functions, trigonometric function, and the rational functions, are obtained. These methods are more effective and simple than other methods and a number of solutions can be obtained at the same time. The related results are enriched.
\end{abstract}

\section{Introduction}

It has recently become more interesting to obtain exact solutions of nonlinear partial differential equations. These equations are mathematical models of complex physical phenomena that arise in engineering, applied mathematics, chemistry, biology, mechanics, physics, and so forth. Thus, the investigation of the traveling wave solutions to nonlinear evolution equations (NLEEs) plays an important role in mathematical physics. A lot of physical models have supported a wide variety of solitary wave solutions.

The $G^{\prime} / G$-expansion method was proposed by Wang et al. [1], by which a large number of nonlinear evolution equations are studied, such as the KdV equation, the $\mathrm{mKdV}$ equation, the variant Boussinesq equations, and the Hirota-Satsuma equations. Later, the further developed methods named the generalized $G^{\prime} / G$-expansion method, the modified $G^{\prime} / G^{-}$ expansion method, the extended $G^{\prime} / G$-expansion method, and the improved $G^{\prime} / G$-expansion method have been proposed in [2-5], respectively.

In 1995, based on the physical and asymptotic considerations, Fokas [6] derived the following generalized KdV equation:

$$
\begin{gathered}
\eta_{t}+\eta_{x}+\alpha \eta \eta_{x}+\beta \eta_{x x x}+\rho_{1} \alpha^{2} \eta^{2} \eta_{x}+\alpha \beta\left(\rho_{2} \eta \eta_{x x x}+\rho_{3} \eta_{x} \eta_{x x}\right) \\
+\rho_{4} \alpha^{3} \eta^{3} \eta_{x}+\alpha^{2} \beta\left(\rho_{5} \eta^{2} \eta_{x x x}+\rho_{6} \eta \eta_{x} \eta_{x x}+\rho_{7} \eta_{x}^{3}\right)=0,
\end{gathered}
$$

which is an important water wave model, where $\alpha=3 \mathrm{~A} / 2$, $\beta=B / 6, \rho_{1}=-1 / 6, \rho_{6}=5 / 3, \rho_{3}=23 / 6, \rho_{4}=1 / 8, \rho_{5}=7 / 18$, $\rho_{6}=79 / 36$, and $\rho_{7}=45 / 36$. Regarding the $\rho_{1}, \rho_{2}, \rho_{3}, \rho_{4}, \rho_{5}$, $\rho_{6}$, and $\rho_{7}$ as free parameters and using the $\widetilde{\rho}_{4}$ to replace the $\rho_{4} \alpha^{2}$, (1) becomes the following PDE:

$$
\begin{aligned}
u_{t} & +u_{x}+\alpha u u_{x}+\beta u_{x x x}+\rho_{1} \alpha^{2} u^{2} u_{x} \\
& +\alpha \beta\left(\rho_{2} u u_{x x x}+\rho_{3} u_{x} \eta_{x x}\right)+\tilde{\rho}_{4} \alpha u^{3} u_{x} \\
& +\alpha^{2} \beta\left(\rho_{5} u^{2} u_{x x x}+\rho_{6} u u_{x} u_{x x}+\rho_{7} \eta_{x}^{3}\right)=0,
\end{aligned}
$$

which is given by Tzirtzilakis et al. in [7]. They called it highorder wave equation of KdV type. Just as Tzirtzilakis et al. [8] said, these two equations are both water wave equations of KdV type, which are more physically and practically meaningful.

Assuming that the waves are unidirectional and neglecting terms of $O\left(\alpha^{2}, \beta^{3}, \alpha \beta\right)$, (1) can be reduced to the classical $\mathrm{KdV}$ equation:

$$
\eta_{t}+\eta_{x}+\alpha \eta \eta_{x}+\beta \eta_{x x x}=0 .
$$

The integrability and solutions of KdV equation and KdV type equation are studied by a lot of researchers [9-13]. If we neglect the highest order infinitesimal term of $O\left(\alpha^{2} \beta\right)$, 
then (1) can be reduced to a new generalized KdV equation as follows:

$$
\begin{aligned}
\eta_{t} & +\eta_{x}+\alpha \eta \eta_{x}+\beta \eta_{x x x}+\rho_{1} \alpha^{2} \eta^{2} \eta_{x} \\
& +\alpha \beta\left(\rho_{2} \eta \eta_{x x x}+\rho_{3} \eta_{x} \eta_{x x}\right)+\rho_{4} \alpha^{3} \eta^{3} \eta_{x}=0 .
\end{aligned}
$$

In fact, (4) is another special case of (1) for $\rho_{5}=\rho_{6}=\rho_{7}=$ 0 ; it is also third order approximate equation of KdV type. Of course, on describing dynamical behaviors of water waves, (4) is only a rough approximative model of (1).

Equation (1) is studied by many researchers and some useful results are obtained when $\rho_{i}$ takes special values. However, by using the current methods, we cannot obtain exact solutions of (1) in universal conditions. Therefore, the investigation of exact solutions of (4) is necessary and important. Equation (4) is perhaps not integrable. But it would be interesting to check its asymptotic integrability [14].

Equation (4) is studied by Wu et al. in [15] using the integral bifurcation method and some exact solutions in parameter form are given by He et al. in [16] using extended $F$-expansion method. In this paper, regarding the $\rho_{i}(i=$ $1,2,3,4)$ as free parameters and by using $G^{\prime} / G$-expansion, the improved $G^{\prime} / G$-expansion method, and extended $G^{\prime} / G$ expansion method, we will investigate exact traveling wave solutions of (4).

The organization of the paper is as follows. In Section 2, a brief account of the $G^{\prime} / G$-expansion and its variants, that is, the generalized, the improved, and the extended versions, for finding the traveling wave solutions of nonlinear equations is given. In Section 3, we will study (4) by these methods. Finally, conclusions are given in Section 4.

\section{Description of the Methods}

\subsection{The $G^{\prime} / G$-Expansion Method and Improved Method}

Step 1. Consider a general nonlinear PDE in the form

$$
\left(u, u_{x}, u_{t}, u_{x x}, u_{x t}, \ldots\right)=0 .
$$

Using $u(x, t)=U(\xi), \xi=\alpha x+\beta t$, we can rewrite (5) as the following nonlinear ODE:

$$
\left(U, U^{\prime}, U^{\prime \prime}, \ldots\right)=0
$$

where the prime denotes differentiation with respect to $\xi$.

Step 2. Suppose that the solution of ODE (6) can be written as follows:

$$
U(\xi)=\sum_{i=0}^{n} a_{i}\left(\frac{G^{\prime}}{G}\right)^{i}+\sum_{j=1}^{n} b_{j}\left(\frac{G^{\prime}}{G}\right)^{-j}
$$

or

$$
U(\xi)=\sum_{i=0}^{n} a_{i}\left(\frac{G^{\prime}}{G+\sigma G^{\prime}}\right)^{i}+\sum_{j=1}^{n} b_{j}\left(\frac{G^{\prime}}{G+\sigma G^{\prime}}\right)^{-j}
$$

where $\sigma, a_{i}, b_{j}$ are constants to be determined later, $n$ is a positive integer, and $G=G(\xi)$ satisfies the following second order linear ordinary differential equation:

$$
G^{\prime \prime}+\lambda G^{\prime}+\mu G=0,
$$

where $\lambda, \mu$ are real constants. The general solutions of (9) can be listed as follows.

When $\Delta=\lambda^{2}-4 \mu>0$, we obtain the hyperbolic function solution of (9):

$$
G(\xi)=e^{-(\lambda / 2) \xi}\left(A_{1} \cosh \left(\frac{\sqrt{\Delta}}{2} \xi\right)+A_{2} \sinh \left(\frac{\sqrt{\Delta}}{2} \xi\right)\right) .
$$

When $\Delta=\lambda^{2}-4 \mu<0$, we obtain the trigonometric function solution of (9):

$$
G(\xi)=e^{-(\lambda / 2) \xi}\left(A_{1} \cos \left(\frac{\sqrt{-\Delta}}{2} \xi\right)+A_{2} \sin \left(\frac{\sqrt{-\Delta}}{2} \xi\right)\right) .
$$

When $\Delta=\lambda^{2}-4 \mu=0$, we obtain the solution of (9):

$$
G(\xi)=e^{-(\lambda / 2) \xi}\left(A_{1}+A_{2} \xi\right)
$$

where $A_{1}$ and $A_{2}$ are arbitrary constants.

Step 3. Determine the positive integer $n$ by balancing the highest order derivatives and nonlinear terms in (6).

Step 4. Substituting (7) or (8) along with (9) into (6) and then setting all the coefficients of $\left(G^{\prime} / G\right)^{k}(k=1,2, \ldots)$ of the resulting system's numerator to zero yield a set of overdetermined nonlinear algebraic equations for $c$ and $a_{i}, b_{j}$.

Step 5. Assuming that the constants $c$ and $a_{i}, b_{i}$ can be obtained by solving the algebraic equations in Step 4, then substituting these constants and the known general solutions of (9) into (7) or (8), we can obtain the explicit solutions of (5) immediately.

2.2. The Generalized $G^{\prime} / G$-Expansion Method. In generalized version, one makes an ansatz for the solution $U(\xi)$ as

$$
U(\xi)=\sum_{i=0}^{n} a_{i}\left(\frac{G^{\prime}}{G}\right)^{i}+\sum_{j=1}^{n} b_{j}\left(\frac{G^{\prime}}{G}\right)^{-j},
$$

where $G=G(\xi)$ satisfies the following equation:

$$
\left(G^{\prime}\right)^{2}=h_{0}+h_{1} G+h_{2} G^{2}+h_{3} G^{3}+h_{4} G^{4},
$$

where $h_{0}, h_{1}, h_{2}, h_{3}$, and $h_{4}$ are the arbitrary constants to be determined later and $a_{n} b_{n} \neq 0$. Substituting (13) into (5) and using (14), we obtain a polynomial in $G^{i}, G^{\prime} G^{i}(i=1,2, \ldots)$. Equating each coefficient of the resulting polynomials to zero yields a set of algebraic equations for $a_{i}, b_{j}$, and $h_{i}$. Substituting $a_{i}, b_{i}$ and the general solutions of (12) appending on $h_{i}(i=$ $0,1, \ldots, 4)$ into (13), we obtain many new traveling wave solutions of the nonlinear PDE (5). 
2.3. The Extended $G^{\prime} / G$-Expansion Method. In the extended form of this method, the solution $U(\xi)$ of (6) can be expressed as

$$
\begin{aligned}
U(\xi)= & a_{0}+\sum_{i=1}^{n}\left(a_{i}\left(\frac{G^{\prime}}{G}\right)^{i}\right. \\
& \left.+b_{i}\left(\frac{G^{\prime}}{G}\right)^{i-1} \sqrt{\sigma\left(1+\frac{1}{\mu}\left(\frac{G^{\prime}}{G}\right)^{2}\right)}\right),
\end{aligned}
$$

where $a_{0}, a_{i}$, and $b_{i}(i=1,2, \ldots, n)$ are constants to be determined later, $\sigma= \pm 1, n$ is a positive integer, and $G=G(\xi)$ satisfies the following second order linear ODE:

$$
G^{\prime \prime}+\mu G=0,
$$

where $\mu$ is a constant. Substituting (15) into (6) and using (16) and collecting all terms with the same order of $\left(G^{\prime} / G\right)^{k}$ and $\left(G^{\prime} / G\right)^{k} \sqrt{\sigma\left(1+(1 / \mu)\left(G^{\prime} / G\right)^{2}\right)}$ together and then equating each coefficient of the resulting polynomial to zero yield a set of algebraic equations for $\mu, a_{0}, a_{i}, b_{i}(i=1, \ldots, n)$. On solving these algebraic equations, we obtain the values of the constants $\mu, a_{0}, a_{i}, b_{i}(i=1, \ldots, n)$ and then substituting these constants and the known general solutions of (16), which can be got by setting $\lambda=0$ in (10)-(12), into (15), we obtain the explicit solutions of nonlinear differential equation (5).

After the brief description of the methods, we now apply these methods for solving the general KdV equation (4).

\section{Exact Solutions of (4)}

Making a transformation $\eta(t, x)=\phi(\xi)$ with $\xi=x-c t$, (5) can be reduced to the following ODE:

$$
\begin{aligned}
& -c \phi^{\prime}+\phi^{\prime}+\alpha \phi \phi^{\prime}+\beta \phi^{\prime \prime \prime}+\rho_{1} \alpha^{2} \phi^{2} \phi^{\prime} \\
& +\alpha \beta\left(\rho_{2} \phi \phi^{\prime \prime \prime}+\rho_{3} \phi^{\prime} \phi^{\prime \prime}\right)+\rho_{4} \alpha^{3} \phi^{3} \phi^{\prime}=0,
\end{aligned}
$$

where $c$ is wave velocity which moves along the direction of $x$-axis and $c \neq 0$. Integrating (17) once and setting the integral constant as $R$ yield

$$
\begin{aligned}
& (1-c) \phi+\frac{1}{2} \alpha \phi^{2}+\beta \phi^{\prime \prime}+\frac{1}{3} \rho_{1} \alpha^{2} \phi^{3} \\
& \quad+\alpha \beta\left(\rho_{2} \phi \phi^{\prime \prime}+\frac{1}{2}\left(\rho_{3}-\rho_{2}\right) \phi^{\prime 2}\right)+\frac{1}{4} \rho_{4} \alpha^{3} \phi^{4}+R=0 .
\end{aligned}
$$

Balancing $\phi \phi^{\prime \prime}$ with $\phi^{4}$ in (18), we find $n+n+2=4 n$, which gives $n=1$.

\subsection{Using $G^{\prime} / G$-Expansion Method and Improved Method}

3.1.1. Using $G^{\prime} / G$-Expansion Method. Suppose that (18) owns the solutions in the form

$$
\phi(\xi)=a_{0}+a_{1} \frac{G^{\prime}}{G}+b_{1}\left(\frac{G^{\prime}}{G}\right)^{-1}
$$

Substituting (19) along with (9) into (18) and then setting all the coefficients of $G^{\prime} / G(k=0,1, \ldots)$ of the resulting system's numerator to zero yield a set of overdetermined nonlinear algebraic equations about $a_{0}, a_{1}, b_{1}, c, \alpha, \beta$, and $\rho_{i}$. Solving the overdetermined algebraic equations, we can obtain the following results:

$$
\begin{gathered}
a_{0}=\frac{\beta}{\alpha}( \pm \sqrt{3 \Delta} \lambda+2 \Delta), \quad a_{1}=0, \quad b_{1}= \pm \frac{2 \beta \mu}{\alpha} \sqrt{3 \Delta}, \\
c=1+\frac{1}{3} \rho_{3} \beta^{2} \Delta^{2}, \quad \rho_{1}=\frac{1}{2}\left(2 \rho_{2}+\rho_{3}\right)-\frac{1}{2 \beta \Delta}, \\
\rho_{2}=\rho_{2}, \quad \rho_{3}=\rho_{3}, \quad \rho_{4}=-\frac{3 \rho_{2}+\rho_{3}}{6 \beta \Delta},
\end{gathered}
$$

where $\Delta=\lambda^{2}-4 \mu>0$ and $\lambda$ and $\mu$ are arbitrary constants. Consider

$$
\begin{gathered}
a_{0}=\frac{\beta}{\alpha}( \pm \sqrt{3 \Delta} \lambda+2 \Delta), \quad a_{1}= \pm \frac{2 \beta}{\alpha} \sqrt{3 \Delta}, \quad b_{1}=0, \\
c=1+\frac{1}{3} \rho_{3} \beta^{2} \Delta^{2}, \quad \rho_{1}=\frac{1}{2}\left(2 \rho_{2}+\rho_{3}\right)-\frac{1}{2 \beta \Delta}, \\
\rho_{2}=\rho_{2}, \quad \rho_{3}=\rho_{3}, \quad \rho_{4}=-\frac{3 \rho_{2}+\rho_{3}}{6 \beta \Delta},
\end{gathered}
$$

where $\Delta=\lambda^{2}-4 \mu>0$ and $\lambda$ and $\mu$ are arbitrary constants. Consider

$$
\begin{gathered}
a_{0}=\frac{\beta}{\alpha}\left( \pm \sqrt{3 \Delta_{1}} \lambda+2 \Delta_{1}\right), \quad a_{1}= \pm \frac{2 \beta}{\alpha} \sqrt{3 \Delta_{1}}, \\
b_{1}= \pm \frac{2 \beta \mu}{\alpha} \sqrt{3 \Delta_{1}}, \quad c=1+\frac{1}{3} \rho_{3} \beta^{2} \Delta_{1}^{2} \pm 4 \beta^{2} \rho_{3} \lambda \mu \sqrt{3 \Delta_{1}}, \\
\rho_{1}=\frac{1}{2}\left(2 \rho_{2}+\rho_{3}\right)-\frac{1}{2 \beta \Delta_{1}}, \quad \rho_{2}=\rho_{2}, \quad \rho_{3}=\rho_{3}, \\
\rho_{4}=-\frac{3 \rho_{2}+\rho_{3}}{6 \beta \Delta_{1}},
\end{gathered}
$$

where $\Delta_{1}=\lambda^{2}+8 \mu>0$ and $\lambda$ and $\mu$ are arbitrary constants.

Substituting (20) into (19), using solutions of (9), we obtain the following exact solutions of (4).

When $\Delta=\lambda^{2}-4 \mu>0$, we have the hyperbolic function solution as

$$
\begin{aligned}
& \eta(x, t) \\
& =\frac{\beta}{\alpha}( \pm \sqrt{3 \Delta} \lambda+2 \Delta) \pm \frac{2 \beta \mu}{\alpha} \sqrt{3 \Delta}\left(\frac{G^{\prime}}{G}\right)^{-1}
\end{aligned}
$$




$$
\begin{aligned}
= & \frac{\beta}{\alpha}( \pm \sqrt{3 \Delta} \lambda+2 \Delta) \pm \frac{2 \sqrt{3 \Delta} \beta \mu}{\alpha} \\
\times( & \frac{\sqrt{\Delta}}{2} \frac{A_{1} \sinh ((\sqrt{\Delta} / 2) \xi)+A_{2} \cosh ((\sqrt{\Delta} / 2) \xi)}{A_{1} \cosh ((\sqrt{\Delta} / 2) \xi)+A_{2} \sinh ((\sqrt{\Delta} / 2) \xi)} \\
& \left.-\frac{\lambda}{2}\right)^{-1},
\end{aligned}
$$

where $\xi=x-\left(1+(1 / 3) \rho_{3} \beta^{2} \Delta^{2}\right) t, \rho_{1}=(1 / 2)\left(2 \rho_{2}+\rho_{3}\right)-$ $1 / 2 \beta \Delta$, and $\rho_{4}=-\left(3 \rho_{2}+\rho_{3}\right) / 6 \beta \Delta$.

If we set $\lambda=0$ and $A_{1}=0$, solution (23) becomes

$$
\eta(x, t)=-\frac{4 \beta \mu}{\alpha} \pm \frac{4 \sqrt{3} \beta \mu}{\alpha} \tanh (\sqrt{-\mu} \xi),
$$

where $\mu<0, \xi=x-\left(1+(16 / 3) \rho_{3} \beta^{2} \mu^{2}\right) t, \rho_{1}=(1 / 2)\left(2 \rho_{2}+\right.$ $\left.\rho_{3}\right)+1 / 8 \beta \mu$, and $\rho_{4}=\left(3 \rho_{2}+\rho_{3}\right) / 24 \beta \mu$.

Setting $\lambda=0$ and $A_{2}=0$, solution (23) becomes

$$
\eta(x, t)=-\frac{4 \beta \mu}{\alpha} \pm \frac{4 \sqrt{3} \beta \mu}{\alpha} \operatorname{coth}(\sqrt{-\mu} \xi)
$$

where $\mu<0, \xi=x-\left(1+(16 / 3) \rho_{3} \beta^{2} \mu^{2}\right) t, \rho_{1}=(1 / 2)\left(2 \rho_{2}+\right.$ $\left.\rho_{3}\right)+1 / 8 \beta \mu$, and $\rho_{4}=\left(3 \rho_{2}+\rho_{3}\right) / 24 \beta \mu$.

Substituting (21) into (19), using solutions of (9), we obtain the following exact solutions of (4).

When $\Delta=\lambda^{2}-4 \mu>0$, we have the hyperbolic function solution as

$$
\begin{aligned}
\eta(x, t) & \\
= & \frac{\beta}{\alpha}( \pm \sqrt{3 \Delta} \lambda+2 \Delta) \pm \frac{2 \beta \mu}{\alpha} \sqrt{3 \Delta}\left(\frac{G^{\prime}}{G}\right)^{-1} \\
= & \frac{\beta}{\alpha}( \pm \sqrt{3 \Delta} \lambda+2 \Delta) \pm \frac{2 \sqrt{3 \Delta} \beta \mu}{\alpha} \\
& \times\left(\frac{\sqrt{\Delta}}{2} \frac{A_{1} \sinh ((\sqrt{\Delta} / 2) \xi)+A_{2} \cosh ((\sqrt{\Delta} / 2) \xi)}{A_{1} \cosh ((\sqrt{\Delta} / 2) \xi)+A_{2} \sinh ((\sqrt{\Delta} / 2) \xi)}\right. \\
& \left.-\frac{\lambda}{2}\right),
\end{aligned}
$$

where $\xi=x-\left(1+(1 / 3) \rho_{3} \beta^{2} \Delta^{2}\right) t, \rho_{1}=(1 / 2)\left(2 \rho_{2}+\rho_{3}\right)-$ $1 / 2 \beta \Delta$, and $\rho_{4}=-\left(3 \rho_{2}+\rho_{3}\right) / 6 \beta \Delta$.

Substituting (22) into (19), using solutions of (9), we obtain the following exact solutions of (4).
When $\Delta=\lambda^{2}-4 \mu>0$, we have the hyperbolic function solution as

$$
\begin{aligned}
\eta(x, t) & \\
= & \frac{\beta}{\alpha}\left( \pm \sqrt{3 \Delta_{1}} \lambda+2 \Delta_{1}\right) \pm \frac{2 \beta}{\alpha} \sqrt{3 \Delta_{1}}\left(\frac{G^{\prime}}{G} \pm \mu \frac{G}{G^{\prime}}\right) \\
= & \frac{\beta}{\alpha}\left( \pm \sqrt{3 \Delta_{1}} \lambda+2 \Delta_{1}\right) \pm \frac{2 \sqrt{3 \Delta_{1}} \beta}{\alpha} \\
& \times\left(\frac{\sqrt{\Delta}}{2} \frac{A_{1} \sinh ((\sqrt{\Delta} / 2) \xi)+A_{2} \cosh ((\sqrt{\Delta} / 2) \xi)}{A_{1} \cosh ((\sqrt{\Delta} / 2) \xi)+A_{2} \sinh ((\sqrt{\Delta} / 2) \xi)}\right. \\
& \left.-\frac{\lambda}{2}\right) \pm \frac{2 \sqrt{3 \Delta_{1}} \beta \mu}{\alpha} \\
\times & \left(\frac{\sqrt{\Delta}}{2} \frac{A_{1} \sinh ((\sqrt{\Delta} / 2) \xi)+A_{2} \cosh ((\sqrt{\Delta} / 2) \xi)}{A_{1} \cosh ((\sqrt{\Delta} / 2) \xi)+A_{2} \sinh ((\sqrt{\Delta} / 2) \xi)}\right. \\
& \left.-\frac{\lambda}{2}\right)^{-1},
\end{aligned}
$$

where $\xi=x-\left(1+(1 / 3) \rho_{3} \beta^{2} \Delta_{1}^{2} \pm 4 \beta^{2} \rho_{3} \lambda \mu \sqrt{3 \Delta_{1}}\right) t, \rho_{1}=$ $(1 / 2)\left(2 \rho_{2}+\rho_{3}\right)-1 / 2 \beta \Delta_{1}$, and $\rho_{4}=-\left(3 \rho_{2}+\rho_{3}\right) / 6 \beta \Delta_{1}$.

When $\Delta=\lambda^{2}-4 \mu<0$, we have the trigonometric function solution as

$\eta(x, t)$

$$
\begin{aligned}
= & \frac{\beta}{\alpha}\left( \pm \sqrt{3 \Delta_{1}} \lambda+2 \Delta_{1}\right) \pm \frac{2 \beta}{\alpha} \sqrt{3 \Delta_{1}}\left(\frac{G^{\prime}}{G} \pm \mu \frac{G}{G^{\prime}}\right) \\
= & \frac{\beta}{\alpha}\left( \pm \sqrt{3 \Delta_{1}} \lambda+2 \Delta_{1}\right) \pm \frac{2 \sqrt{3 \Delta_{1}} \beta}{\alpha} \\
& \times\left(\frac{\sqrt{-\Delta}}{2} \frac{-A_{1} \sin ((\sqrt{-\Delta} / 2) \xi)+A_{2} \cos ((\sqrt{-\Delta} / 2) \xi)}{A_{1} \cos ((\sqrt{-\Delta} / 2) \xi)+A_{2} \sin ((\sqrt{-\Delta} / 2) \xi)}\right. \\
& \left.-\frac{\lambda}{2}\right) \pm \frac{2 \sqrt{3 \Delta_{1}} \beta \mu}{\alpha} \\
& \times\left(\frac{\sqrt{-\Delta}}{2} \frac{-A_{1} \sin ((\sqrt{-\Delta} / 2) \xi)+A_{2} \cos ((\sqrt{-\Delta} / 2) \xi)}{A_{1} \cos ((\sqrt{-\Delta} / 2) \xi)+A_{2} \sin ((\sqrt{-\Delta} / 2) \xi)}\right. \\
& \left.-\frac{\lambda}{2}\right)^{-1},
\end{aligned}
$$

where $\xi=x-\left(1+(1 / 3) \rho_{3} \beta^{2} \Delta_{1}^{2}\right) t, \rho_{1}=(1 / 2)\left(2 \rho_{2}+\rho_{3}\right)-$ $1 / 2 \beta \Delta_{1}$, and $\rho_{4}=-\left(3 \rho_{2}+\rho_{3}\right) / 6 \beta \Delta_{1}$.

Setting $\lambda=0$ and $A_{1}=0$, solution (28) becomes

$$
\eta(x, t)=\frac{8 \beta \mu}{\alpha} \pm \frac{4 \sqrt{6} \beta \mu}{\alpha}(\cot (\sqrt{\mu} \xi)+\tan (\sqrt{\mu} \xi)),
$$


where $\mu<0, \xi=x-\left(1+(64 / 3) \rho_{3} \beta^{2} \mu^{2}\right) t, \rho_{1}=(1 / 2)\left(2 \rho_{2}+\right.$ $\left.\rho_{3}\right)-1 / 16 \beta \mu$, and $\rho_{4}=-\left(3 \rho_{2}+\rho_{3}\right) / 48 \beta \mu$.

When $\Delta=\lambda^{2}-4 \mu=0$, we have the rational function solution as

$$
\begin{aligned}
\eta(x, t)= & \frac{\beta}{\alpha}\left( \pm \sqrt{3 \Delta_{1}} \lambda+2 \Delta_{1}\right) \pm \frac{2 \beta}{\alpha} \sqrt{3 \Delta_{1}}\left(\frac{G^{\prime}}{G} \pm \mu \frac{G}{G^{\prime}}\right) \\
= & \frac{\beta}{\alpha}\left( \pm \sqrt{3 \Delta_{1}} \lambda+2 \Delta_{1}\right) \\
& \pm \frac{2 \sqrt{3 \Delta_{1}} \beta}{\alpha}\left(-\frac{\lambda}{2}+\frac{A_{2}}{A_{2} \xi+A_{1}}\right) \\
& \pm \frac{2 \sqrt{3 \Delta_{1}} \beta \mu}{\alpha}\left(-\frac{\lambda}{2}+\frac{A_{2}}{A_{2} \xi+A_{1}}\right)^{-1},
\end{aligned}
$$

where $\xi=x-\left(1+(1 / 3) \rho_{3} \beta^{2} \Delta_{1}^{2}\right) t, \rho_{1}=(1 / 2)\left(2 \rho_{2}+\rho_{3}\right)-$ $1 / 2 \beta \Delta_{1}$, and $\rho_{4}=-\left(3 \rho_{2}+\rho_{3}\right) / 6 \beta \Delta_{1}$.

3.1.2. Using Improved $G^{\prime} / G$-Expansion Method. Suppose that (18) owns the solutions in the form

$$
\phi(\xi)=a_{0}+a_{1} \frac{G^{\prime}}{G+\sigma G^{\prime}}+b_{1}\left(\frac{G^{\prime}}{G+\sigma G^{\prime}}\right)^{-1} .
$$

Substituting (31) along with (9) into (18) and then setting all the coefficients of $G^{\prime} / G(k=0,1, \ldots)$ of the resulting system's numerator to zero yield a set of overdetermined nonlinear algebraic equations about $a_{0}, a_{1}, b_{1}, c, \alpha, \beta$, and $\rho_{i}$. Solving the overdetermined algebraic equations, we can obtain the following results:

$$
\begin{gathered}
a_{0}=\frac{\beta}{\alpha}(\mp 2 \mu \sigma \sqrt{3 \Delta} \pm \lambda \sqrt{3 \Delta}+\Delta), \quad a_{1}=0, \\
b_{1}= \pm \frac{2 \beta \mu}{\alpha} \sqrt{3 \Delta}, \quad c=1+\frac{1}{3} \rho_{3} \beta^{2} \Delta^{2}, \\
\rho_{1}=\frac{1}{2}\left(2 \rho_{2}+\rho_{3}\right)-\frac{1}{2 \beta \Delta}, \quad \rho_{2}=\rho_{2}, \quad \rho_{3}=\rho_{3}, \\
\rho_{4}=-\frac{3 \rho_{2}+\rho_{3}}{6 \beta \Delta},
\end{gathered}
$$

where $\Delta=\lambda^{2}-4 \mu>0$ and $\lambda$ and $\mu$ are arbitrary constants. Consider

$$
\begin{gathered}
a_{0}=-\frac{\beta}{\alpha}(\mp 2 \mu \sigma \sqrt{3 \Delta} \pm \lambda \sqrt{3 \Delta}-\Delta), \\
a_{1}= \pm \frac{2 \beta}{\alpha} \sqrt{3 \Delta}\left(-\mu \sigma^{2}+\lambda \sigma-1\right), \\
c=1-\beta^{2} \Delta^{2}\left(2 \beta \lambda^{2} \rho_{4}-8 \beta \mu \rho_{4}+\rho_{2}\right), \\
b_{1}=0, \quad \rho_{2}=\rho_{2}, \quad \rho_{4}=\rho_{4},
\end{gathered}
$$

$$
\begin{gathered}
\rho_{1}=-\frac{1}{2}\left(6 \beta \lambda^{2} \rho_{4}-24 \beta \mu \rho_{4}+\rho_{2}\right)-\frac{1}{2 \beta \Delta}, \\
\rho_{3}=-6 \beta \rho_{4}\left(\lambda^{2}-4 \mu\right)-3 \rho_{2},
\end{gathered}
$$

where $\Delta=\lambda^{2}-4 \mu>0$ and $\lambda$ and $\mu$ are arbitrary constants.

Comparing (32) and (33) with (20) and (21), respectively, we can find that their structures are the same. Therefore, we do not discuss them in detail.

Remark 1. When $\sigma=0$, the improved $G^{\prime} / G$-expansion method is equal to the old one. However, because of the complication of the computation, the improved method usually cannot obtain solutions which can be got by the old method, such as (22) in our work.

3.2. Using Extended $G^{\prime} / G$-Expansion Method. Suppose that (18) owns the solutions in the form

$$
\phi(\xi)=a_{0}+a_{1} \frac{G^{\prime}}{G}+b_{1} \sqrt{\sigma\left(1+\frac{1}{\mu}\left(\frac{G^{\prime}}{G}\right)^{2}\right)}
$$

where $a_{0}, a_{1}$, and $b_{1}$ are constants to be determined later, $\sigma=$ $\pm 1, n$ is a positive integer, and $G=G(\xi)$ satisfies the second order linear ODE (14).

Substituting (34) along with (16) into (18) and then setting all the coefficients of $\left(G^{\prime} / G\right)^{k}$ and $\left(G^{\prime} / G\right)^{k} \sqrt{\sigma\left(1+(1 / \mu)\left(G^{\prime} / G\right)^{2}\right)}(k=0,1, \ldots)$ of the resulting system to zero yield a set of overdetermined nonlinear algebraic equations about $a_{0}, a_{1}, b_{1}, c, \alpha, \beta$, and $\rho_{i}$. Solving the overdetermined algebraic equations, we can obtain the following results:

$$
\begin{gathered}
a_{0}=\frac{2 \beta \mu}{\alpha}, \quad a_{1}=0, \quad b_{1}= \pm \frac{2 \beta \mu}{\alpha} \sqrt{\frac{6}{\sigma}} \\
c=32 \beta^{3} \mu^{3} \rho_{4}+8 \beta^{2} \mu^{2} \rho_{1}+2 \beta \mu+1, \\
\rho_{1}=\rho_{1}, \quad \rho_{2}=-12 \beta \mu \rho_{4}-2 \rho_{1}-\frac{1}{2 \beta \mu}, \\
\rho_{3}=24 \beta \mu \rho_{4}+6 \rho_{1}+\frac{3}{2 \beta \mu}, \quad \rho_{4}=\rho_{4},
\end{gathered}
$$

where $\sigma>0$. Consider

$$
\begin{gathered}
a_{0}=-\frac{4 \beta \mu}{\alpha}, \quad a_{1}= \pm \frac{4 \beta}{\alpha} \sqrt{-3 \mu}, \quad b_{1}=0, \\
c=128 \beta^{3} \mu^{3} \rho_{4}-16 \beta^{2} \mu^{2} \rho_{2}+1, \\
\rho_{1}=12 \beta \mu \rho_{4}-\frac{\rho_{2}}{2}+\frac{1}{8 \beta \mu}, \quad \rho_{2}=\rho_{2}, \\
\rho_{3}=24 \beta \mu \rho_{4}-3 \rho_{2}, \quad \rho_{4}=\rho_{4},
\end{gathered}
$$


where $\mu<0$. Consider

$$
\begin{gathered}
a_{0}=-\frac{\beta \mu}{\alpha}, \quad a_{1}= \pm \frac{\beta}{\alpha} \sqrt{-3 \mu}, \quad b_{1}= \pm \frac{\beta \mu}{\alpha} \sqrt{-\frac{3}{\sigma}} \\
c=-4 \beta^{3} \mu^{3} \rho_{4}+2 \beta^{2} \mu^{2} \rho_{1}-\beta \mu+1 \\
\rho_{1}=\rho_{1}, \quad \rho_{2}=6 \beta \mu \rho_{4}-2 \rho_{1}+\frac{1}{\mu \beta} \\
\rho_{3}=-12 \beta \mu \rho_{4}+6 \rho_{1}-\frac{3}{\mu \beta}, \quad \rho_{4}=\rho_{4}
\end{gathered}
$$

where $\mu<0$ and $\sigma<0$.

Using (35) and the general solutions of (16) which can be obtained by setting $\lambda=0$ in (10)-(12), we can find the following traveling wave solutions of (4).

When $\mu<0$ and $\sigma=1$, we have the hyperbolic function solution as

$\eta(x, t)$

$$
\begin{aligned}
& =a_{0}+b_{1} \sqrt{\sigma\left(1+\frac{1}{\mu}\left(\frac{G^{\prime}}{G}\right)^{2}\right)} \\
& =\frac{2 \mu \beta}{\alpha} \\
& \quad \times(1 \pm \sqrt{6} \\
& \left.\quad \times \sqrt{1-\left(\frac{A_{1} \sinh (\sqrt{-\mu} \xi)+A_{2} \cosh (\sqrt{-\mu} \xi)}{A_{1} \cosh (\sqrt{-\mu} \xi)+A_{2} \sinh (\sqrt{-\mu} \xi)}\right)^{2}}\right),
\end{aligned}
$$

where $\xi=x-\left(32 \beta^{3} \mu^{3} \rho_{4}+8 \beta^{2} \mu^{2} \rho_{1}+2 \beta \mu+1\right) t, \rho_{2}=-12 \beta \mu \rho_{4}-$ $2 \rho_{1}-1 / 2 \beta \mu$, and $\rho_{3}=24 \beta \mu \rho_{4}+6 \rho_{1}+3 / 2 \beta \mu$.

Setting $A_{1}=0,(38)$ becomes

$$
\eta(x, t)=\frac{2 \mu \beta}{\alpha}\left(1 \pm \sqrt{6} \sqrt{1-\operatorname{coth}^{2}(\sqrt{-\mu} \xi)}\right) .
$$

Setting $A_{2}=0$, (38) becomes

$$
\begin{aligned}
\eta(x, t) & =\frac{2 \mu \beta}{\alpha}\left(1 \pm \sqrt{6} \sqrt{1-\tanh ^{2}(\sqrt{-\mu} \xi)}\right) \\
& =\frac{2 \mu \beta}{\alpha}(1 \pm \sqrt{6} \operatorname{sech}(\sqrt{-\mu} \xi)) .
\end{aligned}
$$

When $\mu>0$ and $\sigma=1$, we have the hyperbolic function solution as

$$
\begin{aligned}
& \eta(x, t) \\
& =a_{0}+b_{1} \sqrt{\sigma\left(1+\frac{1}{\mu}\left(\frac{G^{\prime}}{G}\right)^{2}\right)} \\
& =\frac{2 \mu \beta}{\alpha} \\
& \quad \times\left(1 \pm \sqrt{6} \sqrt{1+\left(\frac{-A_{1} \sin (\sqrt{\mu} \xi)+A_{2} \cos (\sqrt{\mu} \xi)}{A_{1} \cos (\sqrt{\mu} \xi)+A_{2} \sin (\sqrt{\mu} \xi)}\right)}\right),
\end{aligned}
$$

where $\xi=x-\left(32 \beta^{3} \mu^{3} \rho_{4}+8 \beta^{2} \mu^{2} \rho_{1}+2 \beta \mu+1\right) t, \rho_{2}=-12 \beta \mu \rho_{4}-$ $2 \rho_{1}-1 / 2 \beta \mu$, and $\rho_{3}=24 \beta \mu \rho_{4}+6 \rho_{1}+3 / 2 \beta \mu$.

Setting $A_{1}=0$, (41) becomes

$$
\eta(x, t)=\frac{2 \mu \beta}{\alpha}(1 \pm \sqrt{6} \sec (\sqrt{\mu} \xi)) .
$$

Setting $A_{2}=0$, (41) becomes

$$
\eta(x, t)=\frac{2 \mu \beta}{\alpha}(1 \pm \sqrt{6} \csc (\sqrt{\mu} \xi)) .
$$

Using (36) and the general solutions of (16), we can obtain the traveling wave solutions of (4). However, in (36) $b_{1}=$ 0 . The result is similar to (21) obtained by $G^{\prime} / G$-expansion method; therefore, we omit it.

Using (37) and the general solutions of (16), we can obtain the following traveling wave solutions of (4).

When $\mu<0$ and $\sigma=-1$, we have the hyperbolic function solution as

$$
\begin{aligned}
\eta(x, t)= & -\frac{\mu \beta}{\alpha} \\
& \mp \frac{\sqrt{3} \mu \beta}{\alpha} \frac{A_{1} \sinh (\sqrt{-\mu} \xi)+A_{2} \cosh (\sqrt{-\mu} \xi)}{A_{1} \cosh (\sqrt{-\mu} \xi)+A_{2} \sinh (\sqrt{-\mu} \xi)} \\
& \pm \frac{\sqrt{3} \mu \beta}{\alpha} \\
& \times \sqrt{-1+\left(\frac{A_{1} \sinh (\sqrt{-\mu} \xi)+A_{2} \cosh (\sqrt{-\mu} \xi)}{A_{1} \cosh (\sqrt{-\mu} \xi)+A_{2} \sinh (\sqrt{-\mu} \xi)}\right)^{2}},
\end{aligned}
$$

where $\xi=x-\left(-4 \beta^{3} \mu^{3} \rho_{4}+2 \beta^{2} \mu^{2} \rho_{1}-\beta \mu+1\right) t, \rho_{2}=6 \beta \mu \rho_{4}-$ $2 \rho_{1}+1 / \mu \beta$, and $\rho_{3}=-12 \beta \mu \rho_{4}+6 \rho_{1}-3 / \mu \beta$.

Setting $A_{1}=0$, (44) becomes

$$
\eta(x, t)=-\frac{\mu \beta}{\alpha}(1 \pm \sqrt{3} \operatorname{coth}(\sqrt{-\mu} \xi) \mp \sqrt{3} \operatorname{csch}(\sqrt{-\mu} \xi))
$$


Setting $A_{2}=0$, (44) becomes

$$
\begin{aligned}
& \eta(x, t) \\
& =-\frac{\mu \beta}{\alpha} \\
& \quad \times\left(1 \pm \sqrt{3} \tanh (\sqrt{-\mu} \xi) \mp \sqrt{3} \sqrt{-1+\tanh ^{2}(\sqrt{-\mu} \xi)}\right) .
\end{aligned}
$$

Remark 2. $\sigma$ can be a nonzero constant.

3.3. Using General $G^{\prime} / G$-Expansion Method. Suppose that (18) owns the solutions in the form

$$
\phi(\xi)=a_{0}+a_{1} \frac{G^{\prime}}{G}+b_{1}\left(\frac{G^{\prime}}{G}\right)^{-1} ;
$$

in this case, $G=G(\xi)$ satisfies the Jacobi elliptic equation (14). In order to find new type of solutions, we just consider the case of $h_{1}=h_{3}=0$.

Substituting (47) along with (14) into (18) and then setting all the coefficients of $G^{i}, G^{\prime} G^{i}(i=1,2, \ldots)$ of the resulting system's numerator to zero yield a set of overdetermined nonlinear algebraic equations about $a_{0}, a_{1}, b_{1}, c$, and $\rho_{i}$. Solving the overdetermined algebraic equations, we can obtain the following results:

$$
\begin{gathered}
a_{0}=\frac{4 \beta h_{2}}{\alpha}, \quad a_{1}=0, \quad b_{1}= \pm \frac{4 \beta}{\alpha} \sqrt{3 h_{2}\left(h_{2}^{2}-4 h_{2} h_{4}\right)}, \\
c=1-16 \beta^{2} h_{2}^{2}\left(8 \beta h_{2} \rho_{4}+\rho_{2}\right), \\
\rho_{1}=-12 \beta h_{2} \rho_{4}-\frac{\rho_{2}}{2}-\frac{1}{8 \beta h_{2}}, \quad \rho_{2}=\rho_{2} \\
\rho_{3}=-24 \beta h_{2} \rho_{4}-3 \rho_{2}, \quad \rho_{4}=\rho_{4},
\end{gathered}
$$

where $3 h_{2}\left(h_{2}^{2}-4 h_{2} h_{4}\right)>0$, and

$$
\begin{gathered}
a_{0}=\frac{4 \beta h_{2}}{\alpha}, \quad a_{1}= \pm \frac{4 \beta}{\alpha} \sqrt{3 h_{2}}, \quad b_{1}=0, \\
c=1-16 \beta^{2} h_{2}^{2}\left(8 \beta h_{2} \rho_{4}+\rho_{2}\right), \\
\rho_{1}=-12 \beta h_{2} \rho_{4}-\frac{\rho_{2}}{2}-\frac{1}{8 \beta h_{2}}, \quad \rho_{2}=\rho_{2}, \\
\rho_{3}=-24 \beta h_{2} \rho_{4}-3 \rho_{2}, \quad \rho_{4}=\rho_{4},
\end{gathered}
$$

where $h_{2}>0$.

When $h_{1}=h_{3}=0$, the general elliptic equation (14) is reduced to the auxiliary ordinary equation

$$
G^{\prime}(\xi)^{2}=h_{0}+h_{2} G^{2}(\xi)+h_{4} G^{4}(\xi) .
$$

The solutions of (50) are given in Table 1. Substituting (48) and (49) into (47), making use of Table 1, many exact solutions of (4) can be obtained. For simplicity, we just give out one case in Table 1; the other cases can be discussed similarly.
When $h_{0}=m^{2}-1, h_{2}=2-m^{2}$, and $h_{4}=-1$, the solution of $(50)$ is $G(\xi)=\operatorname{dn}(\xi, m)$. We can obtain the following solutions of (4).

From (48), one has

$$
\begin{aligned}
\eta(x, t)= & a_{0}+b_{1} \frac{G}{G^{\prime}} \\
= & \frac{4 \beta h_{2}}{\alpha} \pm \frac{4 \beta}{\alpha} \sqrt{3 h_{2}\left(h_{2}^{2}-4 h_{2} h_{4}\right)} \frac{\mathrm{dn}(\xi, m)}{\operatorname{cn}(\xi, m) \operatorname{sn}(\xi, m)} \\
= & \frac{4 \beta\left(2-m^{2}\right)}{\alpha} \\
& \pm \frac{4 \beta \sqrt{3\left(2-m^{2}\right)}}{\alpha} \frac{\mathrm{dn}(\xi, m)}{\operatorname{cn}(\xi, m) \operatorname{sn}(\xi, m)},
\end{aligned}
$$

where $\xi=x-\left(1+16 \beta^{2}\left(m^{2}-2\right)^{2}\left(8 \beta m^{2} \rho_{4}-16 \beta \rho_{4}-\rho_{2}\right)\right) t$, $\rho_{1}=12 \beta m^{2} \rho_{4}-24 \beta \rho_{4}-\rho_{2} / 2-1 / 8 \beta\left(m^{2}-2\right)$, and $\rho_{3}=$ $24 \rho_{4}\left(m^{2}-2\right) \beta-3 \rho_{2}$.

When $m \rightarrow 1, \operatorname{dn}(\xi, m) \rightarrow \operatorname{sech}(\xi), \operatorname{cn}(\xi, m) \rightarrow$ $\operatorname{sech}(\xi)$, and $\operatorname{sn}(\xi, m) \rightarrow \tanh (\xi),(51)$ becomes

$$
\eta(x, t)=\frac{4 \beta}{\alpha}(1 \pm \sqrt{3} \operatorname{coth}(\xi))
$$

where $\xi=x-\left(1-16 \beta^{2}\left(8 \beta \rho_{4}+\rho_{2}\right)\right) t, \rho_{1}=-12 \beta \rho_{4}-\rho_{2} / 2-1 / 8 \beta$, and $\rho_{3}=-24 \beta \rho_{4}-3 \rho_{2}$.

From (49), one has

$$
\begin{aligned}
\eta(x, t)= & a_{0}+a_{1} \frac{G^{\prime}}{G} \\
= & \frac{4 \beta h_{2}}{\alpha} \pm \frac{4 \beta}{\alpha} \sqrt{3 h_{2}} \frac{\mathrm{cn}(\xi, m) \operatorname{sn}(\xi, m)}{\operatorname{dn}(\xi, m)} \\
= & \frac{4 \beta\left(2-m^{2}\right)}{\alpha} \\
& \pm \frac{4 \beta m^{2} \sqrt{3\left(2-m^{2}\right)}}{\alpha} \frac{\mathrm{cn}(\xi, m) \operatorname{sn}(\xi, m)}{\operatorname{dn}(\xi, m)}
\end{aligned}
$$

where $\xi=x-\left(1+16 \beta^{2}\left(m^{2}-2\right)^{2}\left(8 \beta m^{2} \rho_{4}-16 \beta \rho_{4}-\rho_{2}\right)\right) t, \rho_{1}=$ $12 \beta m^{2} \rho_{4}-24 \beta \rho_{4}-\rho_{2} / 2-1 / 8 \beta\left(m^{2}-2\right)$, and $\rho_{3}=24 \rho_{4}\left(m^{2}-\right.$ 2) $\beta-3 \rho_{2}$.

When $m \rightarrow 1, \operatorname{dn}(\xi, m) \rightarrow \operatorname{sech}(\xi),(53)$ becomes

$$
\eta(x, t)=\frac{4 \beta}{\alpha}(1 \pm \sqrt{3} \tanh (\xi))
$$

where $\xi=x-\left(1-16 \beta^{2}\left(8 \beta \rho_{4}+\rho_{2}\right)\right) t, \rho_{1}=-12 \beta \rho_{4}-\rho_{2} / 2-$ $1 / 8 \beta$, and $\rho_{3}=-24 \beta \rho_{4}-3 \rho_{2}$.

Remark 3. Besides the case of $h_{1}=h_{3}=0$, we can also get other cases which will deduce similar solutions obtained before. Therefore, we omit them for simplicity. 
TABLE 1: Solutions of $G(\xi)$ in $G^{\prime 2}=h_{0}+h_{2} G^{2}+h_{4} G^{4}$.

\begin{tabular}{|c|c|c|c|c|}
\hline Case & $h_{0}$ & $h_{2}$ & $h_{4}$ & $G(\xi)$ \\
\hline 1 & 1 & $-\left(m^{2}+1\right)$ & $m^{2}$ & $\operatorname{sn}(\xi), \operatorname{cd}(\xi)$ \\
\hline 2 & $1-m^{2}$ & $m^{2}-1$ & $-m^{2}$ & $\operatorname{cn}(\xi)$ \\
\hline 3 & $m^{2}-1$ & $2-m^{2}$ & -1 & $\operatorname{dn}(\xi)$ \\
\hline 4 & $m^{2}$ & $-\left(m^{2}+1\right)$ & 1 & $\mathrm{~ns}(\xi), \mathrm{dc}(\xi)$ \\
\hline 5 & $-m^{2}$ & $2 m^{2}-1$ & $1-m^{2}$ & $\mathrm{nc}(\xi)$ \\
\hline 6 & -1 & $2-m^{2}$ & $m^{2}-1$ & $\operatorname{nd}(\xi)$ \\
\hline 7 & 1 & $2-m^{2}$ & $1-m^{2}$ & $\mathrm{sc}(\xi)$ \\
\hline 8 & 1 & $2 m^{2}-1$ & $-m^{2}\left(1-m^{2}\right)$ & $\operatorname{sd}(\xi)$ \\
\hline 9 & $1-m^{2}$ & $2-m^{2}$ & 1 & $\operatorname{cs}(\xi)$ \\
\hline 10 & $-m^{2}\left(1-m^{2}\right)$ & $2 m^{2}-1$ & 1 & $\operatorname{sd}(\xi)$ \\
\hline 11 & $1 / 4$ & $\left(1-2 m^{2}\right) / 2$ & $1 / 4$ & $\mathrm{~ns}(\xi) \pm \operatorname{cs}(\xi)$ \\
\hline 12 & $\left(1-m^{2}\right) / 4$ & $\left(1+m^{2}\right) / 2$ & $\left(1-m^{2}\right) / 4$ & $\mathrm{nc}(\xi) \pm \mathrm{sc}(\xi)$ \\
\hline 13 & $m^{2} / 4$ & $\left(m^{2}-2\right) / 2$ & $1 / 4$ & $\mathrm{~ns}(\xi) \pm \mathrm{ds}(\xi)$ \\
\hline 14 & $m^{2} / 4$ & $\left(m^{2}-2\right) / 2$ & $m^{2} / 4$ & $\operatorname{sn}(\xi) \pm i \operatorname{cn}(\xi)$ \\
\hline
\end{tabular}

\section{Conclusions}

The investigation of the exact solutions of (4) is meaningful and important. However, (4) is just studied by Wu et al. [15] using the integral bifurcation method and He et al. [16] using extended $F$-expansion method. In our work, (4) is studied by multiple $G^{\prime} / G$-expansion method and some new exact solutions expressed by Jacobi elliptic function, hyperbolic function, trigonometric function, and rational function are obtained. We believe the results we obtained are useful in describing related physical phenomena. The correctness of all the solutions is verified by substituting them into original equation (4). Comparing with [15] and [16], it is easy to see that the solutions obtained in our work are more general which contain many free parameters and our method is more straightforward. The related results are enriched.

\section{Conflict of Interests}

The authors declare that there is no conflict of interests regarding the publication of this paper.

\section{Acknowledgments}

This research is supported by the Natural Science Foundation of China (nos. 11161020 and 11361023) and the Natural Science Foundation of Yunnan (2013FZ117).

\section{References}

[1] M. Wang, X. Li, and J. Zhang, "The $G^{\prime} / G$-expansion method and travelling wave solutions of nonlinear evolution equations in mathematical physics," Physics Letters A, vol. 372, no. 4, pp. 417-423, 2008.

[2] S. Zhang, J. L. Tong, and W. Wang, "A generalized $G^{\prime} / G$ expansion method for the $\mathrm{mKdV}$ equation with variable coefficients," Physics Letters A, vol. 372, pp. 2254-2257, 2008.
[3] Y.-B. Zhou and C. Li, "Application of modified $G^{\prime} / G$-expansion method to traveling wave solutions for Whitham-Broer-Kauplike equations," Communications in Theoretical Physics, vol. 51, no. 4, pp. 664-670, 2009.

[4] S. Guo and Y. Zhou, "The extended $G^{\prime} / G$-expansion method and its applications to the Whitham-Broer-Kaup-like equations and coupled Hirota-Satsuma KdV equations," Applied Mathematics and Computation, vol. 215, no. 9, pp. 3214-3221, 2010.

[5] S. Guo, Y. Zhou, and C. Zhao, "The improved $G^{\prime} / G$-expansion method and its applications to the Broer-Kaup equations and approximate long water wave equations," Applied Mathematics and Computation, vol. 216, no. 7, pp. 1965-1971, 2010.

[6] A. S. Fokas, "On a class of physically important integrable equations," Physica D, vol. 87, no. 1-4, pp. 145-150, 1995.

[7] E. Tzirtzilakis, V. Marinakis, C. Apokis, and T. Bountis, "Soliton-like solutions of higher order wave equations of the Korteweg-de Vries type," Journal of Mathematical Physics, vol. 43, no. 12, pp. 6151-6161, 2002.

[8] E. Tzirtzilakis, M. Xenos, V. Marinakis, and T. C. Bountis, "Interactions and stability of solitary waves in shallow water," Chaos, Solitons \& Fractals, vol. 14, no. 1, pp. 87-95, 2002.

[9] V. E. Zakharov and A. B. Shabat, "A scheme for integrating the nonlinear equations of mathematical physics by the method of the inverse scattering problem. I," Functional Analysis and Its Applications, vol. 8, no. 3, pp. 226-235, 1974.

[10] V. E. Zaharov and A. B. Shabat, "Integration of the nonlinear equations of mathematical physics by the method of the inverse scattering problem. II," Functional Analysis and Its Applications, vol. 13, no. 3, pp. 166-174, 1979.

[11] M. Ruggieri, "Kink solutions for a class of generalized dissipative equations," Abstract and Applied Analysis, vol. 2012, Article ID 237135, 7 pages, 2012.

[12] M. Ruggieri and A. Valenti, "Approximate symmetries in nonlinear viscoelastic media," Boundary Value Problems, vol. 2013, article 143, pp. 1-8, 2013.

[13] M. Ruggieri and M. P. Speciale, "Similarity reduction and closed form solutions for a model derived from two-layer fluids," Advances in Difference Equations, vol. 2013, no. 1, article 355, 2013. 
[14] Y. Kodama and A. V. Mikhailov, "Obstacles to asymptotic integrability," in Algebraic Aspects of Integrability, pp. 173-204, Birkhäuser, Basel, Switzerland, 1996, edited by I. M. Gelfand and A. Fokas.

[15] X. Wu, W. Rui, and X. Hong, "A generalized KdV equation of neglecting the highest-order infinitesimal term and its exact traveling wave solutions," Abstract and Applied Analysis, vol. 2013, Article ID 656297, 15 pages, 2013.

[16] Y. He, Y.-M. Zhao, and Y. Long, "New exact solutions for a higher-order wave equation of KdV type using extended Fexpansion method," Mathematical Problems in Engineering, vol. 2013, Article ID 128970, 8 pages, 2013. 


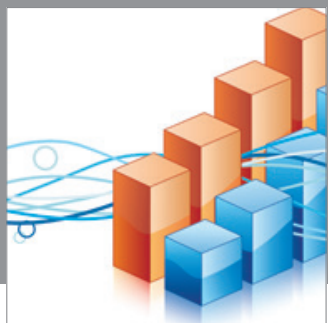

Advances in

Operations Research

mansans

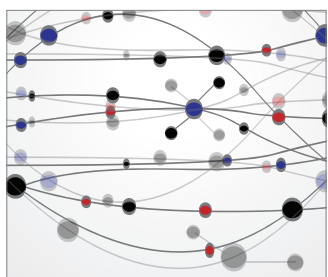

The Scientific World Journal
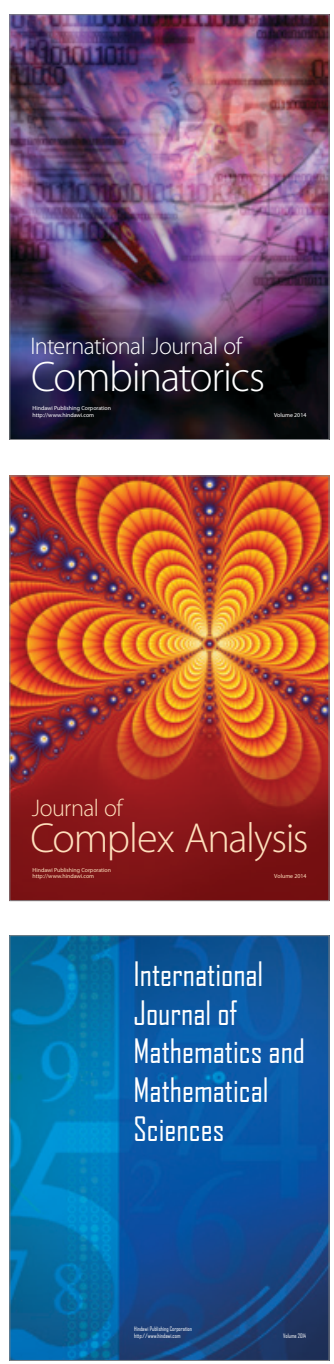
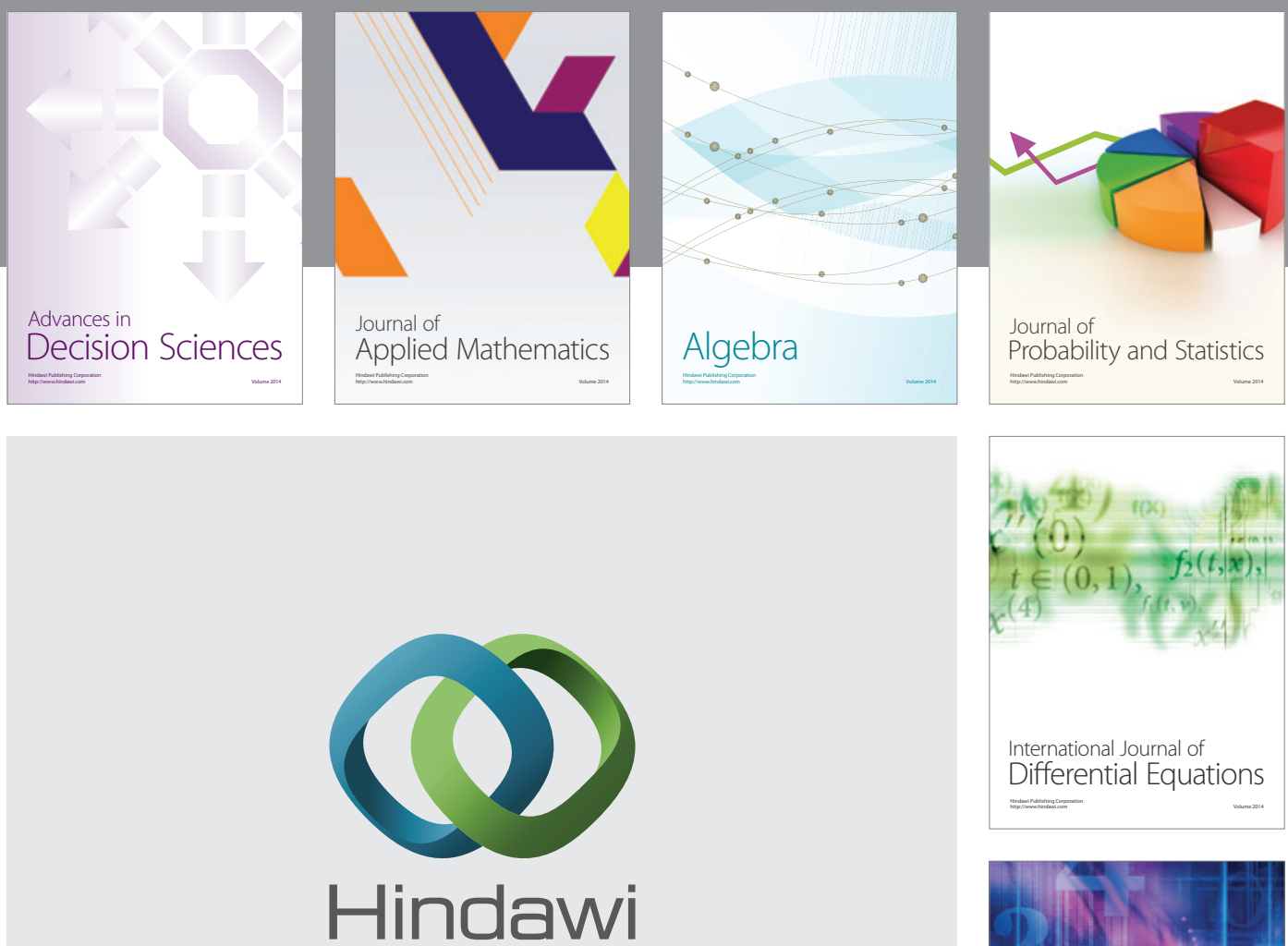

Submit your manuscripts at http://www.hindawi.com
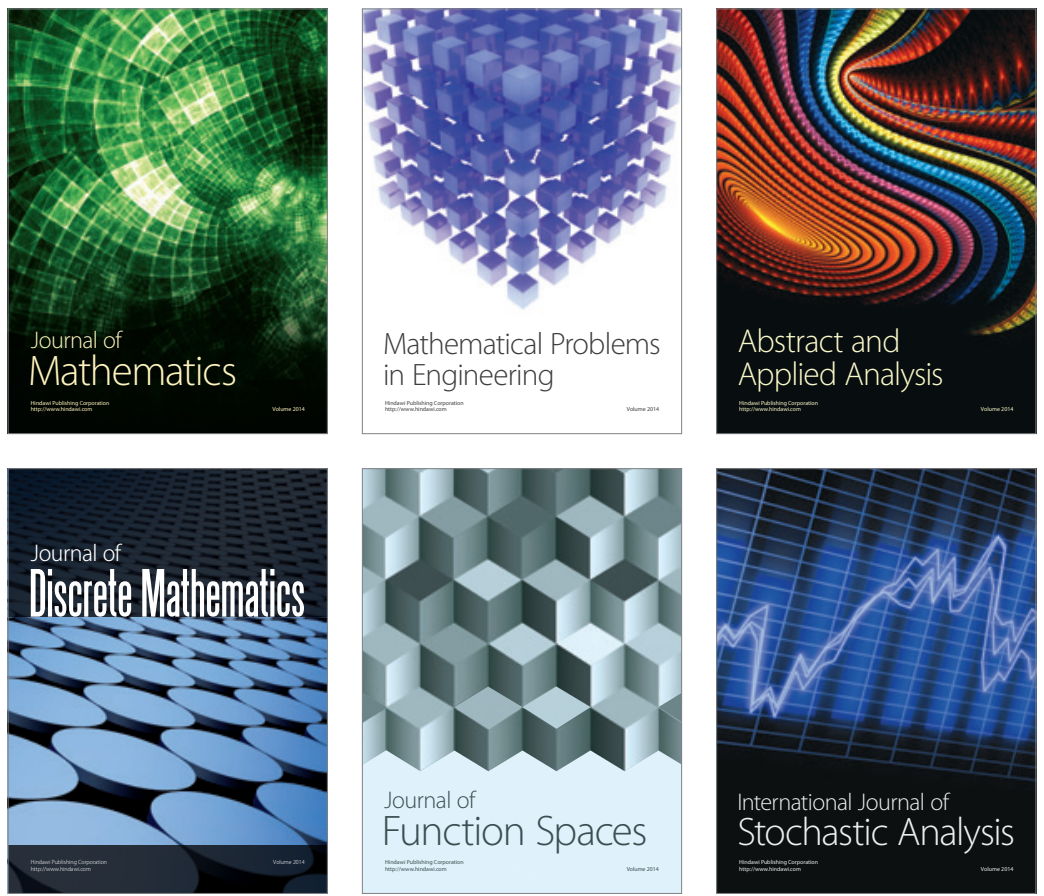

Journal of

Function Spaces

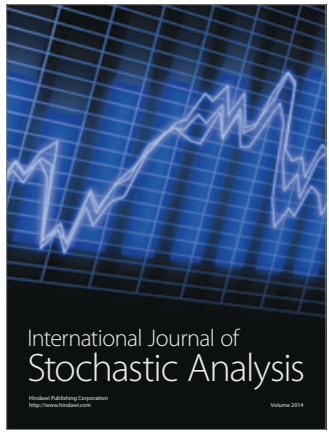

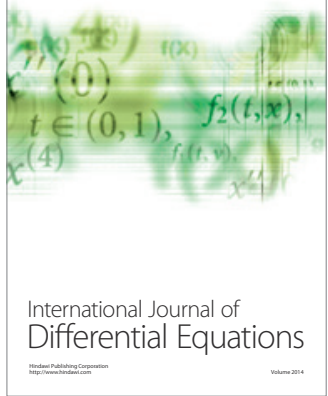
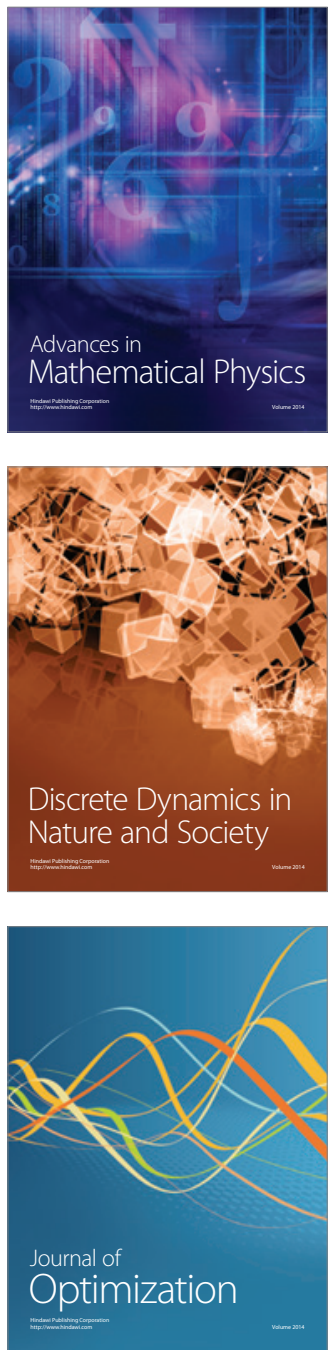